\title{
PRE-LABOUR RUPTURE OF MEMBRANES: A CASE REPORT, MANAGEMENT \& LITERATURE REVIEW
}

\author{
Kimera Lukanga charles ${ }^{*}$, Linda Ndeshipandula Lukolo² \\ $1 *$ Lecturer and HoD department of Obstetrics and Gynaecology, School of Medicine, University of Namibia \\ ${ }^{2}$ Lecturer Department of Family Medicine, School of Medicine, University of Namibia
}

\section{*Correspondent author's}

Email:lkimera@unam.na

\begin{abstract}
: -
Rupture of membranes during pregnancy before labour begins, referred to as premature rupture of membranes (PROM), is a known risk factor for subsequent maternal and foetal/neonatal infection although pre-existing infection may at times be the cause of membrane rupture. This infection may lead to poor pregnancy outcome if appropriate management is not instituted in time. Recognition of the period of rupture, by proper history taking, is of paramount importance as further management of the mother will depend on this. This paper reviews a case report of a mismanaged pre-labour rupture of membranes which resulted in loss of a foetus/baby in one of the public hospitals.
\end{abstract}

Key wards: Pre-labour Rupture of Membranes, Premature rupture of membranes, PROM, ROM, PPROM, Vaginal examination, Chorioamnionitis,

Words Key: concepts of drugs emergent, new substances psychoactive, club drugs, synthesis drugs, intelligent drugs.

\section{(a) $(\$)$}




\section{CASE SUMMARY}

On 15 $5^{\text {th }}$ June 2016 at 08:00 miss MT presented on maternity ward of public hospital with a term pregnancy (39 week plus 5 days gestation) with a complaint of leakage of amniotic fluid. She was not in labour as she did not have uterine contractions. A diagnosis of premature rupture of membranes (PROM) was made.

On $16^{\text {th }}$ June 2016 at 09:30 (251/2 hours since admission) she started getting one (1) weak contraction lasting 20 seconds in 10 minutes. Her pulse was recorded to be $120 \mathrm{bpm}$ and continued to be high till intervention was made. Her respiratory rate ranged between 20 and 22 per minute at rest.

On 17 th June 2016 at 06:20 Ms MT was in second stage and had spent almost 1 hour bearing down. The midwife informed doctor of a mother with poor maternal effort and the doctor telephonically instructed her (the midwife) to give the mother more time to bear down and only inform him if there was no progress.

One hour later (07:30) the doctor was again called this time to inform him that there is foetal bradycardia (FHR 60$100 \mathrm{bpm})$. The doctor telephonically ordered the midwife to prepare the mother for theatre. The mother was prepared and taken to theatre and theatre notes indicated that a foul smelling fresh still birth was delivered by caesarean section.

The mother had a total number of 11 documented vaginal examinations. There was no documentation of antibiotic administration.

\section{Diagnosis of premature rupture of membranes}

The diagnosis of premature rupture of membranes is based on history physical examination and laboratory investigations. Many women give a history of a gush of fluid from the vagina, followed by continuous leaking. Urinary incontinence or copious vaginal discharge may have a similar presentation and whenever there is doubt investigations to exclude or confirm PROM are warranted.

Physical examination - A speculum vaginal examination will reveal copious fluid pouring into the vagina either spontaneously or on Valsalva maneuver or cough and when this is seen it is confirmation of rupture of membranes. In less overt cases, a nitrazine paper is used to con firm the diagnosis. In case of rupture of membranes the paper turns deep blue when a pooled vaginal fluid is applied indicating an alkaline $\mathrm{pH}$ of the amniotic fluid. False positive results may be due to blood, semen, soap, or infection with Trichomonas or bacterial vaginosis, which can also raise the $\mathrm{pH}$ of the vagina. Another confirmatory test is the presence of arborization (ferning). Here when pooled vaginal fluid from the posterior vaginal fornix is swabbed onto a glass slide and allowed to dry for at least 10 minutes and examined under a microscope it shows a ferning pattern confirmatory to amniotic fluid.

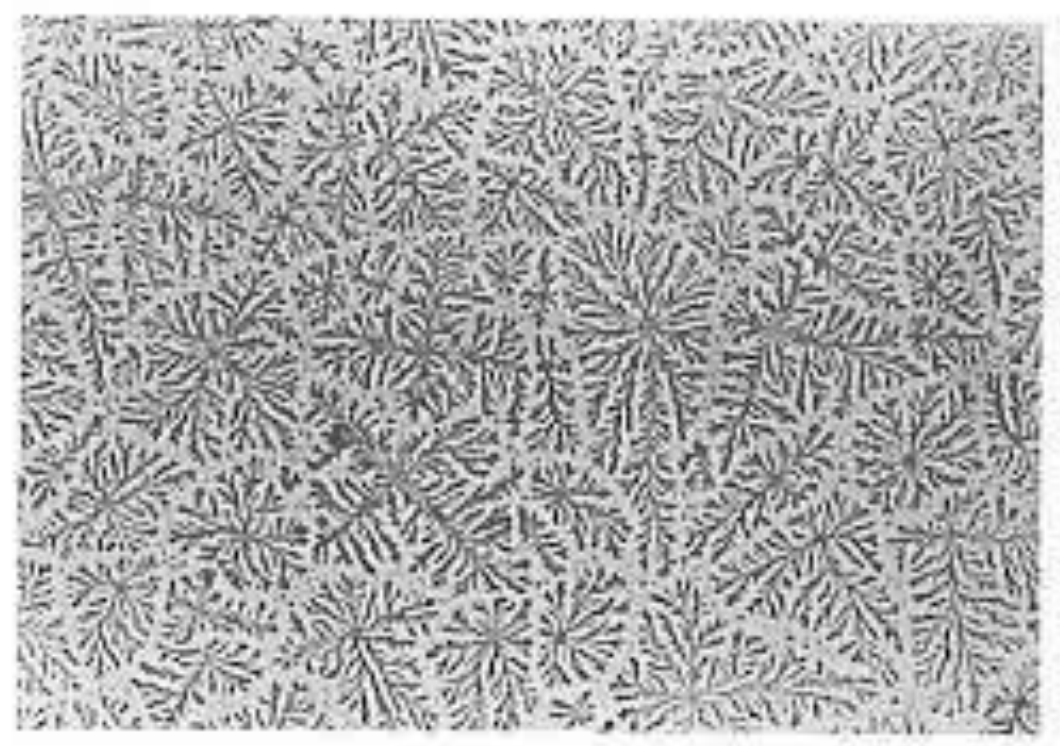

\section{Ferning typically seen when amniotic fluid if smeared on the glass slide and examined under microscope}

\section{Management of Premature (Pre-labour) Ruptured Membranes at Term}

Premature rupture of membranes (PROM) refers to rupture of membranes (ROM) prior to the onset of labor. When ROM occurs after the $37^{\text {th }}$ week of gestation it is referred to as term PROM. Preterm premature rupture of membranes (PPROM), on the other hand, is ROM prior to 37 weeks' gestation. Ms MT had term PROM. Rupture of membranes whether term or preterm is associated with complications depending on the period of time the pregnant woman remains undelivered following membrane rupture. According to the case summary Ms MT spent 45 hour and 20 minutes in hospital before intervention was undertaken to aid her delivery by caesarean section. The period for which membranes were ruptured could not be ascertained, however, since she went to hospital when she was already draining liquor (the admitting staff did not take this history).

Increasing period of time following rupture of the membranes is clearly a risk factor for infection. Research conducted in the 1960s showed that perinatal morbidity and mortality increased significantly if birth did not occur within 24 hours of 
rupture $^{1}$. Ms MT spent over 46 hours with ruptured membranes before she was delivered. Research on factors that are true risks for chorioamnionitis, postpartum endometritis, and neonatal infection suggest that the risk of infection gradually increases with increasing duration of rupture ${ }^{2},{ }_{3}$. It should also be remembered that infection contributes a significant proportion of direct maternal death and that most of this infection is acquired during the peripartum period. Maternal infections around childbirth also have a considerable impact on newborn mortality, and an estimated 1 million newborn deaths are associated with such infections annually ${ }^{4}$.

The risk of chorioamnionitis is about $10 \%$ within the first 24 hours but increase to $40 \%$ after 24 hours ${ }^{5}$. Birth within 24 hours, therefore, is a common management goal when the membranes are ruptured for term pregnancies and those that get rupture after the gestation age at which the foetal lungs are presumed to have matured i.e. after 34 weeks of gestation as there are no added benefits of prolonging the pregnancy in this case. This means that the health personnel need to consider induction of labor in those without obstetric factors that contraindicate a vaginal delivery if labour does not start within 24 hours or caesarean section for those with such obstetric conditions unfavorable for vaginal delivery.

There are factors other than duration of rupture of membranes known to increase risk of infection when membranes are ruptured. One strong predictive factor is the number of vaginal examinations ${ }^{6},{ }^{7}$. The higher the number of vaginal examinations the greater the risk of infection therefore these have to be kept to the minimum. The number of documented vaginal examinations MT had (11) were too many and could have contributed, secondary to prolonged membrane rupture, to the chorioamnionitis and foetal sepsis documented in the patient file, and the resultant perinatal loss. WHO recommends vaginal examination to be performed at 4 hourly intervals for low risk parturient. The recommendation farther states that in situations where there is increased risk of infection such as prolonged rupture of amniotic membranes the frequency and number of vaginal examinations should be restricted ${ }^{8}$. This means that if labour progresses at the slowest acceptable rate, i.e. $1 \mathrm{~cm}$ cervical dilatation per hour, the parturient will get a maximum of 2 vaginal examination from the start of active phase of labour to delivery. Additionally it is advisable to avoid digital vaginal examinations until labour has been established

Use of antibiotics: The Centers for Disease Control and Prevention (CDC) recommends administration of intrapartum chemoprophylaxis to all pregnant women identified as group B streptococcus (GBS) carriers with ruptured membranes. If, however, the woman's GBS status is unknown like in this case, antibiotics for group B streptococcus prevention are recommended when the membranes have been ruptured for 18 hours and the woman has not gone into labour ${ }^{9}$. Antibiotic agents used in the prophylaxis include Ampicillin and erythromycin. A loading dose of $2 \mathrm{gm}$ ampicillin followed by 500mg of erythromycin 6hourly is recommended.

Ms MT wasn't given any antibiotic prophylaxis and even when evidence of infection set in like the high pulse, and the high respiratory rate she was not given antibiotic therapy. The personnel could have under looked these signs and therefore did not consider antibiotic therapy.

\section{Summary and recommendations}

- It is important to always establish the period of rupture of membranes by history taking as this is important to decide on management options.

- Prophylactic antibiotic administration should be initiated immediately in women with term PROM who are culture positive for group B Streptococcus and after 18 hours in those with unknown group B streptococcus status.

- Induction of labor (with oxytocin or prostaglandin E2) in women with term PROM should be considered to reduce the PROM-to-delivery interval and the associated complications

- Digital vaginal examination should be restricted before labour is established and the number of vaginal examinations should be minimized to 4 hourly PVs in active phase of labour.

\section{References}

[1].Lanier LR, Scarbrough RW, Fillingim DW, Baker RE. Incidence of maternal and fetal complications associated with rupture of the membranes before onset of labor. Am J Obstet Gynecol 1965;93:398-404.Bryans CI. Incidence of maternal and fetal complications associated with rupture of the membranes before the onset of labor. Am J Obstet Gynecol 1965;93:403-404.

[2].Soper DE, Mayhall CG, Froggatt JW. Characterization and control of intraamniotic infection in an urban teaching hospital. Am J Obstet Gynecol 1996;175:304-310.

[3].Newton ER, Prihoda TJ, Gibbs RS. Logistic regression analysis of risk factors for intra-amniotic infection. Obstet Gynecol 1989;73:571-575.

[4].Lawn JE, Cousens S, Zupan J. 4 million neonatal deaths: When? Where? Why? The Lancet.365(9462):891900.

[5].Seaward PG, Hannah ME, Myhr TL, et al. International Multicentre Term Prelabor Rupture of Membranes Study: evaluation of predictors of clinical chorioamnionitis and postpartum fever in patients with prelabor rupture of membranes at term. Am J Obstet Gynecol. 1997 Nov. 177(5):1024-9.

[6].Soper DE, Mayhall CG, Dalton HP. Risk factors for intraamniotic infection: A prospective epidemiologic study. Am J Obstet Gynecol 1989;161:562-568.

[7].Seaward PG, Hannah ME, Myhr TL, Farine D, Ohlsson A, Wang EE. International Multicenter Term PROM Study: Evaluation of predictors of neonatal infection in infants born to patient with premature rupture of membranes at term. Am J Obstet Gynecol 1998;179:635-639. 
[8].WHO recommendation on digital vaginal examination at intervals of four hours for routine assessment of active first stage of labour in low-risk women (September 2015). The WHO Reproductive Health Library; Geneva: World Health Organization.

[9].Centers for Disease Control and Prevention. Prevention of perinatal group B streptococcal disease: Revised guidelines from CDC. MMWR 2002;51:RR-11: 1-28. 